\title{
Síntesis hidrotermal de zeolita a partir de ceniza volante tipo F: influencia de la temperatura
}

\author{
Hydrothermal synthesis of zeolite from coal class F fly ash. \\ Influence of temperature
}

\author{
$\underline{\text { S. Goñi }}{ }^{*}$, R. Peña ${ }^{(*)}$, A. Guerrero(*)
}

Recepción/Received: 09-V-2008

Aceptación/Accepted: 20-VIII-2008

Publicado online/Online publishing: 24-II-2010

RESUMEN

En este trabajo se presenta el papel que juega la temperatura durante el tratamiento hidrotermal en medio alcalino para convertir una ceniza volante de bajo contenido en cal (clase F, según la norma ASTM) en zeolita. Durante este tratamiento a la temperatura de $100{ }^{\circ} \mathrm{C}$ se forma zeolita Na-P1 tipo gismondina $\left(\mathrm{Na}_{6} \mathrm{Al}_{6} \mathrm{Si}_{10} \mathrm{O}_{32} \cdot 12 \mathrm{H}_{2} \mathrm{O}\right)$; al elevar la temperatura a $200{ }^{\circ} \mathrm{C}$, dicha zeolita se transforma en zeolita analcima $\mathrm{C}\left(\mathrm{Na}\left(\mathrm{Si}_{2} \mathrm{Al}\right) \mathrm{O}_{6} \mathrm{H}_{2} \mathrm{O}\right)$ y en fase sodalita (1.08 $\left.\mathrm{Na}_{2} \mathrm{O} . \mathrm{Al}_{2} \mathrm{O}_{3} .1 .68 \mathrm{SiO}_{2} \cdot 1.8 \mathrm{H}_{2} \mathrm{O}\right)$ junto con trazas de tobermorita- $11 \AA\left(\mathrm{Ca}_{5}(\mathrm{OH})_{2} \mathrm{Si}_{6} \mathrm{O}_{16} \cdot 4 \mathrm{H}_{2} \mathrm{O}\right)$. A esta temperatura y en estas condiciones se ha conseguido un $100 \%$ de reacción. Un estudio equivalente se ha llevado a cabo empleando agua como medio de referencia. La conversión de ceniza volante en zeolita se ha caracterizado mediante técnicas, como difracción de Rayos X (DRX), espectroscopia infrarroja por transformada de Fourier (FTIR) y análisis térmico (TG/ATD); asimismo los cambios en el área superficial se han llevado a cabo mediante la técnica BET- $\mathrm{N}_{2}$.

Palabras clave: zeolitas, residuos, tratamiento hidrotermal, DRX, análisis térmico.

\section{SUMMARY}

The influence of temperature of alkaline hydrothermal treatment on the conversion in zeolite of Spanish coal low calcium-fly ash (ASTM class $F$ ) is presented in this work. Zeolite Na-P1 gismondine type $\left(\mathrm{Na}_{6} \mathrm{Al}_{6} \mathrm{Si}_{10} \mathrm{O}_{32} .12 \mathrm{H}_{2} \mathrm{O}\right)$ was formed at the temperature of $100^{\circ} \mathrm{C}$, which transformed in zeolite; analcime-C type $\left(\mathrm{Na}\left(\mathrm{Si}_{2} \mathrm{Al}\right) \mathrm{O}_{6} \mathrm{H}_{2} \mathrm{O}\right)$ and sodalite $\left(1.08 \mathrm{Na}_{2} \mathrm{O} . \mathrm{Al}_{2} \mathrm{O}_{3} \cdot 1.68 \mathrm{SiO}_{2} .1 .8 \mathrm{H}_{2} \mathrm{O}\right)$ at $200^{\circ} \mathrm{C}$ together with traces of tobermorite- $11 \AA\left(\mathrm{Ca}_{5}(\mathrm{OH})_{2} \mathrm{Si}_{6} \mathrm{O}_{16}\right.$. $4 \mathrm{H}_{2} \mathrm{O}$ ). At this temperature the $100 \%$ of the fly ash reaction was allowed. An equivalent study was carried out in water as reference. The zeolite conversion of the fly ash was characterized by $X$ ray diffraction $(X R D), F T$ infrared (FTIR) spectroscopy, surface area $\left(B E T-N_{2}\right)$ and thermal analyses

Keywords: zeolites, wastes, hydrothermal treatment, $X R D$, thermal analysis

(*) Instituto de Ciencias de la Construcción Eduardo Torroja (IETcc-CSIC) (Madrid, España). 


\section{INTRODUCCIÓN}

Se están llevando a cabo importantes investigaciones sobre las potenciales aplicaciones del tratamiento hidrotermal $(\mathrm{TH})$ en diferentes tipos de residuos, como son las cenizas volantes de la combustión del carbón y las cenizas volantes procedentes de la incineración de residuos sólidos urbanos. El TH en condiciones de presión aumenta la reactividad de los sólidos y, por lo tanto, la cinética de cualquier proceso. Además, se favorece, desde un punto de vista termodinámico, la formación de fases estables, como es el caso de la fase katoita cúbica $\left(\mathrm{Ca}_{3} \mathrm{Al}_{2}\left(\mathrm{SiO}_{4}\right)(\mathrm{OH})_{8}\right)$, el gel $\mathrm{C}-\mathrm{S}-\mathrm{H}\left(\mathrm{Ca}_{1.5} \mathrm{SiO}_{3.5} . \mathrm{XH}_{2} \mathrm{O}\right)$, a- $\mathrm{C}_{2} \mathrm{SH}$, la tobermorita sustituida por aluminio $\left(\mathrm{Ca}_{5} \mathrm{Si}_{5} \mathrm{Al}(\mathrm{OH}) \mathrm{O}_{17} \cdot 5 \mathrm{H}_{2} \mathrm{O}\right)$ y zeolitas tipo A (hidroxi-sodalita) $\mathrm{Na}_{6}\left[\mathrm{AlSiO}_{4}\right]_{6} \cdot 4 \mathrm{H}_{2} \mathrm{O}$ (1-3). Estas fases pueden actuar tanto como precursores de cementos belíticos reactivos después de su calentamiento (4-6), como de fases inmovilizadoras de especies tóxicas como el Cs, Cd, $\mathrm{Pb}$ y $\mathrm{Cr}(7-8)$.

Este trabajo es parte de un amplio proyecto de investigación relacionado con la formulación de matrices alternativas en base cemento para la inmovilización de residuos líquidos tóxicos-peligrosos en general y radiactivos en particular, en las cuales los productos "zeolíticos" juegan un papel muy importante (9-14).

Se están investigando concretamente, dos tipos de zeolitas: analcima $\left(\mathrm{Na}\left(\mathrm{Si}_{2} \mathrm{Al}\right) \mathrm{O}_{6} \mathrm{H}_{2} \mathrm{O}\right)$ y zeolita $\mathrm{Na}-\mathrm{P} 1$ tipo gismondina $\left(\mathrm{Na}_{6} \mathrm{Al}_{6} \mathrm{Si}_{10} \mathrm{O}_{32} .12 \mathrm{H}_{2} \mathrm{O}\right)$, que han sido sintetizadas en nuestro laboratorio, a partir de cenizas volantes de la combustión del carbón tipo $F$, de acuerdo con trabajos previos llevados a cabo por otros autores (15-17). La capacidad de inmovilización de residuos líquidos con Cs y otros elementos tóxicos de estas zeolitas y matrices base cemento-zeolita será presentado en otras publicaciones.

Weiping Ma y col. (15) en su trabajo sintetizaron zeolita $\mathrm{Na}-\mathrm{P}$ mediante un $\mathrm{TH}$ partiendo de una ceniza volante de la combustión del carbón tipo $\mathrm{F}$ añadiendo cuarzo, empleando disoluciones alcalinas de $\mathrm{NaOH}$ 2,8 $\mathrm{M}$ y $5 \mathrm{M}$ a $100{ }^{\circ} \mathrm{C}$. La zeolita sintetizada mostró alta selectividad por el Cs y el Xr. Querol y col., en sus trabajos (16-17) sintetizaron zeolita Na-P1 mediante un $\mathrm{TH}$ y una ceniza volante de la combustión del carbón tipo $\mathrm{F}$ similar a la empleada en nuestro trabajo. Estos autores usaron una disolución $\mathrm{NaOH} 1 \mathrm{M}$ y una relación líquido/sólido $=18$, con una temperatura de $150^{\circ} \mathrm{C}$ durante $24 \mathrm{~h}$; estos mismos autores vieron que había una transformación de zeolita tipo gismondina en analcime a $200{ }^{\circ} \mathrm{C}$.

En la Tabla 1 se incluye una recopilación de datos tomados de la literatura con el tipo de zeolita obtenida después de un tratamiento en disoluciones alcalinas de $\mathrm{NaOH}$, empleando como materia prima, cenizas volantes

\section{INTRODUCTION}

Considerable research is being conducted on the potential of hydrothermal treatment (HT) for the re-use of a number of types of waste, including the fly ash from steam power plants or municipal solid waste incinerators. At high pressures, HT enhances the reactivity of solids and therefore the kinetics of any process. Moreover, it favours the formation of stable (thermodynamically speaking) phases such as cubic katoite $\left(\mathrm{Ca}_{3} \mathrm{Al}_{2}\left(\mathrm{SiO}_{4}\right)(\mathrm{OH})_{8}\right), \mathrm{C}-\mathrm{S}-\mathrm{H}$ gel $\left(\mathrm{Ca}_{1.5} \mathrm{SiO}_{3.5} \times \mathrm{xH}_{2} \mathrm{O}\right)$, a- $\mathrm{C}_{2} \mathrm{SH}$, aluminium-substituted tobermorite $\left(\mathrm{Ca}_{5} \mathrm{Si}_{5} \mathrm{Al}(\mathrm{OH}) \mathrm{O}_{17} \cdot 5 \mathrm{H}_{2} \mathrm{O}\right)$ and type $A$ zeolites (hydroxy-sodalite) ( $\left.\mathrm{Na}_{6}\left[\mathrm{AlSiO}_{4}\right]{ }_{6} \cdot 4 \mathrm{H}_{2} \mathrm{O}\right)$ (1-3). These phases may act both as reactive belite cement precursors after heating (4-6) and as phases able to immobilize toxic species such as Cs, Cd, $\mathrm{Pb}$ and $\mathrm{Cr}$ (7-8).

This study forms part of a broader research project relating to the formulation of alternative cement-based matrices to immobilize toxic and hazardous liquid waste in general and radioactive waste in particular, an area where "zeolitic" products have a significant role to play (9-14).

More specifically, two types of zeolites are being researched: analcime $\left(\mathrm{Na}\left(\mathrm{Si}_{2} \mathrm{Al}\right) \mathrm{O}_{6} \mathrm{H}_{2} \mathrm{O}\right)$ and zeolite $\mathrm{Na}$ P1 ( $\left.\mathrm{Na}_{6} \mathrm{Al}_{6} \mathrm{Si}_{10} \mathrm{O}_{32} .12 \mathrm{H}_{2} \mathrm{O}\right)$, a gismondine isotype, laboratory-synthesized from class $F$ coal fly ash as described by other authors $\left(\mathrm{Na}\left(\mathrm{Si}_{2} \mathrm{Al}\right)\right.$. The capacity of these zeolites and cement-zeolite base matrices to immobilize Cs-containing liquids will be reported in future papers.

Weiping Ma et al. (15), synthesized zeolite Na-P with HT from quartz-additioned class $F$ fly ash in 2.8- and 5-M alkaline solutions of $\mathrm{NaOH}$ at $100{ }^{\circ} \mathrm{C}$. The zeolite obtained exhibited high selectivity for Cs and Xr. Querol et al. (16-17) synthesized zeolite Na-P1 using HT and a class $F$ coal fly ash similar to the one used here. These authors stored the ash in a $1-\mathrm{M} \mathrm{NaOH}$ solution at a liquid/solid ratio of 18 and a temperature of $150{ }^{\circ} \mathrm{C}$ for 24 hours. These same authors reported the transformation of a gismondine group zeolite into analcime at $200^{\circ} \mathrm{C}$.

Table 1 lists data compiled from the literature on the type of zeolites obtained by treating class F coal fly ash in alkaline $\mathrm{NaOH}$ solutions (15-25). The table shows that the synthesis parameters differed widely. Concentrations 
de la combustión del carbón de tipo F (15-25). Como se puede ver, los parámetros de síntesis son muy diferentes. Así, la concentración empleada de $\mathrm{NaOH}$ varía desde $0,5 \mathrm{M}$ hasta $5 \mathrm{M}$, la temperatura de tratamiento va desde $80^{\circ} \mathrm{C}$ hasta $200^{\circ} \mathrm{C}$; el tiempo de tratamiento desde 3h hasta 7días y la relación líquido/sólido desde 2 a 18. varied from 0.5 to $5 \mathrm{M}$, temperature from 80 to $200{ }^{\circ} \mathrm{C}$, treatment time from $3 \mathrm{~h}$ to 7 days and the liquid/solid ratio from 2 to 18 .

Tabla 1 / Table 1

Síntesis de zeolita a partir de cenizas volantes mediante tratamiento hidrotermal (TH). Hydrothermal (TH) synthesis of zeolite from fly ash.

\begin{tabular}{|c|c|c|c|c|c|c|}
\hline Autores / Authors & $\begin{array}{c}\text { Materiales de } \\
\text { partida / Starting } \\
\text { materials }\end{array}$ & $\begin{array}{l}\text { Tipo de alkali / } \\
\text { Alkali type }\end{array}$ & $\begin{array}{c}\text { Tiempo / } \\
\text { Time }\end{array}$ & $\begin{array}{l}\text { Temperatura / } \\
\text { Temperature }\end{array}$ & $\begin{array}{l}\text { Relación } \\
\text { L/SL/S1 } \\
\text { ratio }\end{array}$ & Producto / Product \\
\hline W. Ma et al. [15] & Class F & $\begin{array}{c}\mathrm{NaOH} \\
2.8 \mathrm{M} \text { or } 5 \mathrm{M}\end{array}$ & $6 \mathrm{~d}$ & $\begin{array}{l}\text { Aging two days at } \\
\text { room temp. }+100^{\circ} \mathrm{C}\end{array}$ & 2.5 & $\begin{array}{c}\mathrm{NaP} \\
\mathrm{Na}_{6}\left[\left(\mathrm{AlO}_{2}\right)_{6}\right. \\
\left.\left.\mathrm{AlO}_{2}\right)_{6}\left(\mathrm{SiO}_{2}\right)_{10}\right] .15 \mathrm{H}_{2} \mathrm{O} \\
\text { (ideal composition) }\end{array}$ \\
\hline Goñi et al. (present paper) & Class F² & $\begin{array}{l}\mathrm{NaOH} \\
1 \mathrm{M}\end{array}$ & $12 \mathrm{~h}$ & $\begin{array}{l}150^{\circ} \mathrm{C} \\
200^{\circ} \mathrm{C}\end{array}$ & 10 & $\begin{array}{c}\mathrm{Na}-\mathrm{P} 1 \text { gismondine } \\
\left(\mathrm{Na}_{6} \mathrm{Al}_{6} \mathrm{Si}_{10} \mathrm{O}_{32} .12 \mathrm{H}_{2} \mathrm{O}\right) \\
\text { Analcime- } \mathrm{C}\left(\mathrm{Na}\left(\mathrm{Si}_{2} \mathrm{Al}\right) \mathrm{O}_{6} \mathrm{H}_{2} \mathrm{O}\right.\end{array}$ \\
\hline Querol et al. [16] & Class $\mathrm{F}^{3}$ & $\begin{array}{l}\mathrm{NaOH} 1 \mathrm{M} \\
\mathrm{NaOH} 1 \mathrm{M}\end{array}$ & $\begin{array}{l}15 \mathrm{~h} \\
15 \mathrm{~h}\end{array}$ & $\begin{array}{l}150^{\circ} \mathrm{C} \\
200^{\circ} \mathrm{C}\end{array}$ & 18 & $\begin{array}{c}\mathrm{P} 1 \mathrm{Na}\left(\mathrm{Na}_{6} \mathrm{Al}_{6} \mathrm{Si}_{10} \mathrm{O}_{32} .12 \mathrm{H}_{2} \mathrm{O}\right) \\
\mathrm{P} 1 \mathrm{Na} \text {, analcime } \\
\left(\mathrm{Na}\left(\mathrm{Si}_{2} \mathrm{All}\right) \mathrm{O}_{6} \mathrm{H}_{2} \mathrm{O}\right)\end{array}$ \\
\hline Querol et al. [17] & $\begin{array}{l}\text { Class F4 } \\
\text { (pilot scale) }\end{array}$ & $\begin{array}{l}\mathrm{NaOH} 1 \mathrm{M} \\
\mathrm{NaOH} 1 \mathrm{M} \\
\mathrm{NaOH} 3 \mathrm{M} \\
\mathrm{NaOH} 5 \mathrm{M}\end{array}$ & $\begin{array}{l}24 \mathrm{~h} \\
24 \mathrm{~h} \\
24 \mathrm{~h} \\
24 \mathrm{~h}\end{array}$ & $\begin{array}{l}150^{\circ} \mathrm{C} \\
200^{\circ} \mathrm{C} \\
150^{\circ} \mathrm{C} \\
200^{\circ} \mathrm{C}\end{array}$ & $\begin{array}{l}18 \\
18 \\
18 \\
18\end{array}$ & $\begin{array}{c}\mathrm{P} 1 \mathrm{Na}\left(\mathrm{Na}_{6} \mathrm{Al}_{6} \mathrm{Si}_{10} \mathrm{O}_{32} .12 \mathrm{H}_{2} \mathrm{O}\right) \\
\text { analcime }\left(\mathrm{Na}\left(\mathrm{Si}_{2} \mathrm{Al}\right) \mathrm{O}_{6} \mathrm{H}_{2} \mathrm{O}\right) \\
\text { hydroxy-sodalite } \\
\left(\mathrm{Na}_{1.08} \mathrm{Al}_{2} \mathrm{Si}_{1.68} \mathrm{O}_{7.44 .1 .8} \mathrm{H}_{2} \mathrm{O}\right) \\
\text { hydroxy-cancrinite } \\
\left(\mathrm{Na}_{14} \mathrm{Al}_{12} \mathrm{Si}_{13} \mathrm{O}_{51} .6 \mathrm{H}_{2} \mathrm{O}\right.\end{array}$ \\
\hline Moreno et al. [18] & $\begin{array}{l}\text { Class F5 (pilot scale) } \\
\text { Class F6 (pilot scale) }\end{array}$ & $\begin{array}{l}\mathrm{NaOH} 2 \mathrm{M} \\
\mathrm{NaOH} 3 \mathrm{M}\end{array}$ & $\begin{array}{l}24 \mathrm{~h} \\
8 \mathrm{~h}\end{array}$ & $\begin{array}{l}150^{\circ} \mathrm{C} \\
125^{\circ} \mathrm{C}\end{array}$ & $\begin{array}{l}2 \\
2\end{array}$ & $\begin{array}{l}\mathrm{P} 1 \mathrm{Na}\left(\mathrm{Na}_{6} \mathrm{Al}_{6} \mathrm{Si}_{10} \mathrm{O}_{32} \cdot 12 \mathrm{H}_{2} \mathrm{O}\right) \\
\mathrm{P} 1 \mathrm{Na}\left(\mathrm{Na}_{6} \mathrm{Al}_{6} \mathrm{Si}_{10} \mathrm{O}_{32} \cdot 12 \mathrm{H}_{2} \mathrm{O}\right)\end{array}$ \\
\hline Moreno et al. [19] & $\begin{array}{l}\text { Silica extracts of } \\
\text { Class } F^{7}\end{array}$ & $\mathrm{NaOH} 2 \mathrm{M}$ & $6 \mathrm{~h}$ & $90^{\circ} \mathrm{C}$ & 3 & Zeolite 4A/X (60/40) \\
\hline Grutzeck and Siemer [20] & $\begin{array}{c}\text { Class F } \\
\text { Sodium } \\
\text { Aluminate slurry }\end{array}$ & $\begin{array}{c}\text { Highly alkaline waste } \\
\text { stream }\end{array}$ & $1,3,7 \mathrm{~d}$ & $\begin{array}{l}80^{\circ} \mathrm{C} \\
130^{\circ} \mathrm{C} \\
180^{\circ} \mathrm{C}\end{array}$ & & $\begin{array}{c}\text { Zeolite } \mathrm{A} \\
\mathrm{P} 1 \mathrm{Na}(\text { gismondene })+ \\
\text { unnamed hydroxysodalite }\end{array}$ \\
\hline La Rosa et al. [21] & Class F & $\begin{array}{c}\mathrm{NaOH} \text { and } \mathrm{KOH} 2.8- \\
5 \mathrm{M}\end{array}$ & & & & $\begin{array}{l}\mathrm{Na}-\mathrm{P} 1\left(\mathrm{Na}_{3} \mathrm{Al}_{3} \mathrm{Si}_{5} \mathrm{O}_{16} \cdot 6 \mathrm{H}_{2} \mathrm{O}\right) \\
\text { and } \mathrm{Y}\left(\mathrm{Na}_{2} \mathrm{Al}_{2} \mathrm{Si}_{4.5} \mathrm{O}_{13} \cdot \mathrm{xH}_{2} \mathrm{O}\right)\end{array}$ \\
\hline Amrhein et al. [22] & Class $\mathrm{F}$ & $\begin{array}{l}\mathrm{NaOH} 0.5 \mathrm{M} \\
\mathrm{NaOH} 3 \mathrm{M}\end{array}$ & Few days & $\begin{array}{c}100^{\circ} \mathrm{C} \\
150-25^{\circ} \mathrm{C}\end{array}$ & 8 & $\stackrel{\mathrm{Na}-\mathrm{P} 1, \mathrm{P}-\mathrm{C}}{X}$ \\
\hline Murayama et al. [23] & Class F & $\begin{array}{l}\mathrm{NaOH} 1 \mathrm{M} \\
\mathrm{NaOH} 4 \mathrm{M}\end{array}$ & $\begin{array}{l}3 \mathrm{~h} \\
3 \mathrm{~h}\end{array}$ & $120^{\circ} \mathrm{C}$ & $\begin{array}{l}4 \\
4\end{array}$ & $\begin{array}{c}\text { Zeolite } \mathrm{P} \\
\text { Hydroxy-sodalite }\end{array}$ \\
\hline Hollman et al. [24] & $\begin{array}{l}\text { Silica extracts } \\
\text { of Class F }\end{array}$ & $\begin{array}{c}\mathrm{NaOH} 2 \mathrm{M} \\
\text { aluminate waste } \\
\text { solution }\end{array}$ & $\begin{array}{l}6 \mathrm{~h} \\
\text { (incub.) } \\
48 \mathrm{~h}\end{array}$ & $\begin{array}{l}90^{\circ} \mathrm{C} \\
90^{\circ} \mathrm{C}\end{array}$ & & $\begin{array}{l}\mathrm{Na}-\mathrm{P} 1 \\
\mathrm{Na}-\mathrm{A} \\
\mathrm{Na}-\mathrm{X}\end{array}$ \\
\hline Tanaka et al. [25] & Class F & $\begin{array}{l}\mathrm{NaOH} 2 \mathrm{M} \\
\mathrm{NaAlO}_{2}\end{array}$ & $72 \mathrm{~h}$ & $85^{\circ} \mathrm{C}$ & & $\begin{array}{l}\mathrm{Na}-\mathrm{A} \\
X\end{array}$ \\
\hline
\end{tabular}

Aquí, este grupo presenta la conversión de una ceniza volante tipo $\mathrm{F}$ en zeolitas, con un tratamiento hidrotermal $(\mathrm{TH})$ en disolución $\mathrm{NaOH} 1 \mathrm{M}$. La influencia de la temperatura se ha abordado desde $50^{\circ} \mathrm{C}$ hasta $200^{\circ} \mathrm{C}$. Un estudio equivalente se ha llevado a cabo empleando el medio agua desionizada como referencia.
This paper describes the hydrothermal conversion of a class $F$ fly ash into zeolite in a $1-\mathrm{M} \mathrm{NaOH}$ solution. The treatment temperature ranged from 50 to $200{ }^{\circ} \mathrm{C}$. A parallel study was conducted using demineralized water as the control medium. 


\section{MATERIALES Y MÉTODOS}

Para este estudio se ha empleado una ceniza volante, suministrada por la central térmica de Soto de Rivera, denominada R3, cuya composición química es principalmente: $48,9 \% \mathrm{SiO}_{2}, 26,8 \% \mathrm{Al}_{2} \mathrm{O}_{3}, 7,5 \% \mathrm{Fe}_{2} \mathrm{O}_{3}$ and 4,6\% $\mathrm{CaO}$. La ceniza se trató hidrotermalmente, sin agitación, en una disolución $1 \mathrm{M} \mathrm{NaOH}$ y agua desmineralizada como referencia. En ambos casos se empleó una relación líquido/sólido de 10, se mantuvo durante $12 \mathrm{~h}$ a diferentes temperaturas: Ta ambiente, $50^{\circ} \mathrm{C}, 100^{\circ} \mathrm{C}, 150^{\circ} \mathrm{C}$ y $200^{\circ} \mathrm{C}$. Cuando finaliza el tratamiento hidrotermal $(\mathrm{TH})$, se filtra y el sólido sin lavar se seca hasta peso constante a $50^{\circ} \mathrm{C}$. El TH ha sido llevado a cabo con un equipo Parr modelo 4761 (300 mL) y un controlador de temperatura PID modelo 4842.

Los estudios de difracción de rayos $\mathrm{X}$ se llevaron a cabo con un equipo Philips PW 1730 con monocromador de grafito y radición de $\mathrm{Cu} \mathrm{K} \alpha_{1}$. Para la caracterización por espectroscopia infrarroja, se empleó un equipo Atimattson Genesis FTIR TM y pastillas de $\mathrm{KBr}$ conteniendo un $0,5 \%$ de muestra. Las medidas de Área Superficial se llevaron a cabo por el método BET de multipunto, con un equipo ASAP 2010, Micromeritics Instrument Corp., Norcross, GA), usando gas $\mathrm{N}_{2}-77 \mathrm{~K}$ como adsorbato; previo al análisis las muestras se desgasificaron a $50^{\circ} \mathrm{C}$ durante $24 \mathrm{~h}$, hasta alcanzar 0,05 $\mu \mathrm{m}$ de $\mathrm{Hg}$. El dato de área superficial se determina a partir de las isotermas de adsorción, usando el método BET [10], en el rango de presiones relativas $\left(\mathrm{p} / \mathrm{p}_{\mathrm{o}}\right)$ de $0,003-0,3$. El análisis térmico se realizó con un equipo Netzsch con analizador simultáneo STA 409 usando $50 \mathrm{mg}$ de muestra y una corriente dinámica de nitrógeno (flujo $=100 \mathrm{~cm}^{3} / \mathrm{min}$ ) y una velocidad de calentamiento de $10^{\circ} \mathrm{C} / \mathrm{min}$.

\section{RESULTADOS Y DISCUSIÓN}

\subsection{Difracción de Rayos $X$ y área superficial BET-N $\mathrm{N}_{2}$}

Los difractogramas de Rayos $\mathrm{X}$ pertenecientes al TH de la ceniza volante (R3) tanto en agua desmineralizada como en disolución alcalina $\mathrm{Na}(\mathrm{OH}) 1 \mathrm{M}$ aparecen en la Figura 1. En la Figura 2 se representan las variaciones del área superficial de la ceniza volante en función de la temperatura del tratamiento hidrotermal en agua y sosa. Como se muestra en esta figura, la ceniza volante de partida tiene las siguientes fases cristalinas: cuarzo $\left(\alpha-\mathrm{SiO}_{2}\right)$, mullita $\left(\mathrm{Al}_{6} \mathrm{Si}_{2} \mathrm{O}_{13}\right)$ y hematita $\left(\alpha-\mathrm{Fe}_{2} \mathrm{O}_{3}\right)$; también presenta un halo amorfo entre 15-35 zona angular $2 \theta$, que corresponde a la parte vítrea de la ceniza volante. La ceniza volante está catalogada como ASTM clase $\mathrm{F}$, de acuerdo con las especificaciones de la norma ENUNE $450\left(\mathrm{SiO}_{2}+\mathrm{Al}_{2} \mathrm{O}_{3}+\mathrm{Fe}_{2} \mathrm{O}_{3}>70 \%\right)$ y bajo contenido en $\mathrm{CaO}$.

\section{EXPERIMENTAL}

The fly ash used in this study, denominated R3, was supplied by the Soto de Ribera coal-fired steam power plant. Its chemical composition was as follows: $48.9 \%$ $\mathrm{SiO}_{2}, 26.8 \% \mathrm{Al}_{2} \mathrm{O}_{3}, 7.5 \% \mathrm{Fe}_{2} \mathrm{O}_{3}$ and $4.6 \% \mathrm{CaO}$. The ash was hydrothermally treated, without stirring, in a 1-M $\mathrm{NaOH}$ solution or demineralized water, used as a control. The liquid/solid ratio was 10 in both cases. The sample solutions were stored for $12 \mathrm{~h}$ at ambient temperature, $50,100,150$ or $200{ }^{\circ} \mathrm{C}$. After the 12-h hydrothermal treatment $(H T)$, the suspension was filtered and the unwashed solid was dried to a constant weight at $50^{\circ} \mathrm{C}$. HT was performed in a Parr $4761(300-\mathrm{ml})$ pressure vessel fitted with a model 8242 PID temperature controller.

The $X$-ray diffraction studies were conducted on a Philips PW $1730 \mathrm{Cu} \mathrm{Ka}_{1}$ diffractor fitted with a graphite monochromator. An ATI Mattson Genesis FTIR spectrophotometer and $\mathrm{KBr}$ pellets containing $0.5 \%$ of sample were used for infrared spectroscopic characterization. Surface area measurements were taken with the multi-point BET method on a Micromeritics Instrument Corp. ASAP 2010 analyzer using $N_{2}-77$ gas as the adsorbate. The samples were degassed prior to analysis for $24 \mathrm{~h}$ at $50{ }^{\circ} \mathrm{C}$ until they reached $0.05 \mu \mathrm{m} \mathrm{Hg}$. Surface area values were determined from adsorption isotherms with the BET method $(10)$ in a relative pressure $\left(p / p_{0}\right)$ range of 0.003-0.3. Thermal analysis was conducted on a Netzsch STA 409 simultaneous analyzer using $50 \mathrm{mg}$ of sample, a dynamic nitrogen flow (flow $=100 \mathrm{~cm}^{3} / \mathrm{min}$ ) and a heating rate of $10^{\circ} \mathrm{C} / \mathrm{min}$.

\section{RESULTS AND DISCUSSION}

\subsection{X-ray diffraction and $B E T-N_{2}$ surface area}

The X-ray diffractograms for fly ash HT (R3) in both demineralized water and alkaline 1-M NaOH are shown in Figure 1. Figure 2 shows the variations in fly ash surface area with hydrothermal treatment temperature in water and caustic soda. As the figure shows, the following phases were present in the initial fly ash: quart $\left(\alpha-\mathrm{SiO}_{2}\right)$, mullite $\left(\mathrm{Al}_{6} \mathrm{Si}_{2} \mathrm{O}_{13}\right)$ and hematite $\left(\alpha-\mathrm{Fe}_{2} \mathrm{O}_{3}\right)$. An amorphous halo was also observed, between 15 and 35 in the $2 \theta$ angular zone, corresponding to the glassy portion of the fly ash. Pursuant to the specifications laid down in European and Spanish standard EN-UNE 450, the ash was classified as ASTM class $\mathrm{F}\left(\mathrm{SiO}_{2}+\mathrm{Al}_{2} \mathrm{O}_{3}+\right.$ $\mathrm{Fe}_{2} \mathrm{O}_{3}>70 \%$ and a low $\mathrm{CaO}$ content). 


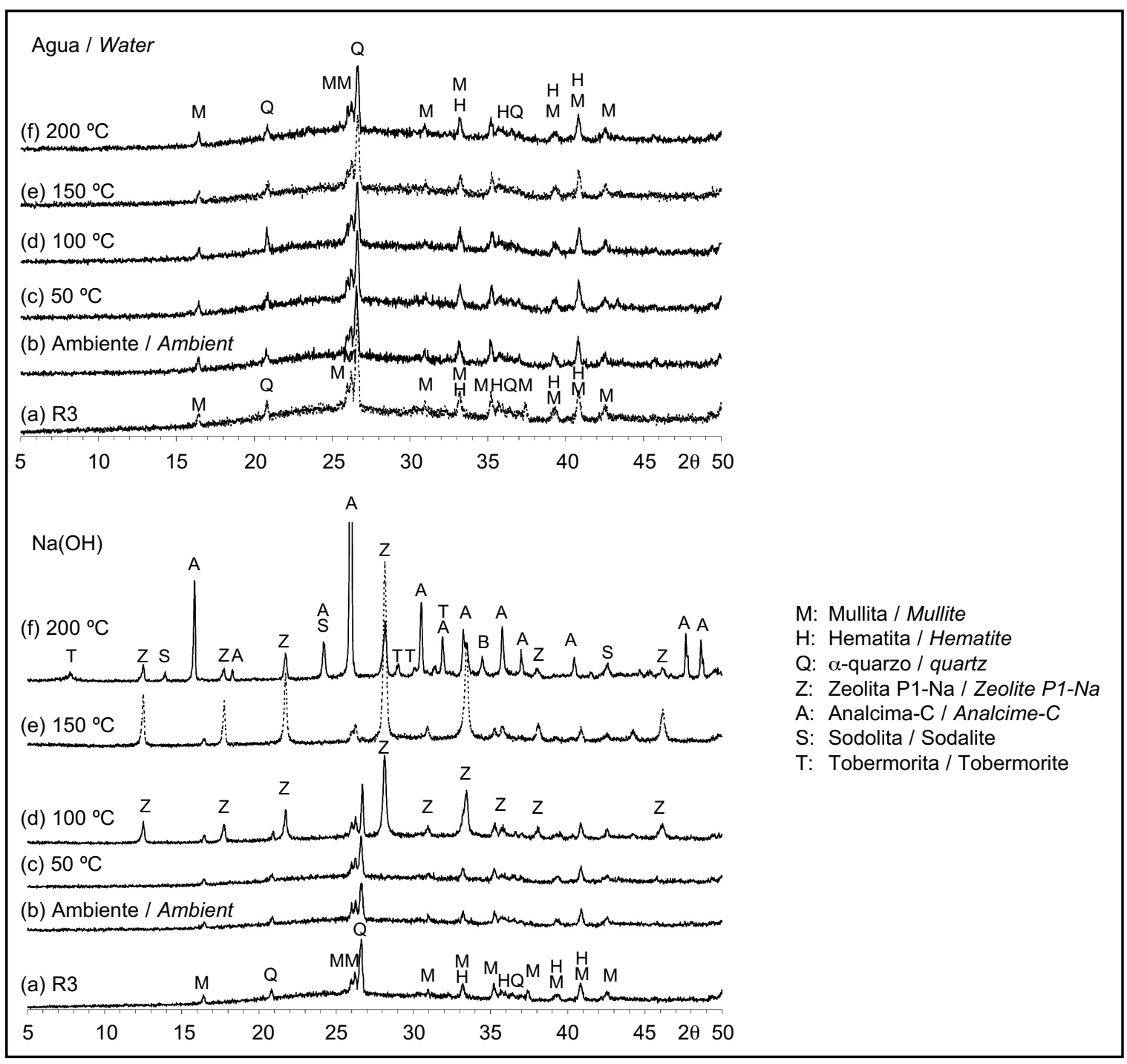

Figura 1. Difractogramas de rayos $\mathrm{X}$ de la ceniza volante como resultado del tratamiento hidrotermal en agua y en disolución $\mathrm{Na}(\mathrm{OH}) 1$. Figure 1. X-ray diffractograms for fly ash after hydrothermal treatment in water and a 1-M NaOH solution.

Con el tratamiento hidrotermal en agua, se observa un aumento en la intensidad de la reflexión correspondiente al cuarzo, especialmente después de $200{ }^{\circ} \mathrm{C}$ (comparar Figura 1 (a) y (f)). El área superficial $\left(B E T-N_{2}\right)$ de la ceniza volante sin tratar R3 aumenta hasta 3 órdenes de magnitud después del tratamiento: desde $0,79 \mathrm{~m}^{2} / \mathrm{g}$ hasta $2,4 \mathrm{~m}^{2} / \mathrm{g}$ a los $200^{\circ} \mathrm{C}$ (ver Figura 2).

Cuando la ceniza volante se somete al $\mathrm{TH}$ en medio $\mathrm{Na}(\mathrm{OH})$, los principales cambios empiezan a observarse después de $100^{\circ} \mathrm{C}$ (Figura 1 (d)- $\mathrm{Na}(\mathrm{OH})$ ), donde se forma la zeolita Na-P1 tipo gismondina $\left(\mathrm{Na}_{6} \mathrm{Al}_{6} \mathrm{Si}_{10} \mathrm{O}_{32} \cdot 12 \mathrm{H}_{2} \mathrm{O}\right)$. En este caso la ceniza volante de partida aumenta hasta 13 veces su área superficial: desde $0,79 \mathrm{~m}^{2} / \mathrm{g}$ hasta 10,2 $\mathrm{m}^{2} / \mathrm{g}$ (Figura 2). A $150^{\circ} \mathrm{C}$ (Figura 1 (e)-Na(OH)), la cantidad de zeolita Na-P1 aumenta, desapareciendo el halo amorfo del $\mathrm{SiO}_{2}$ y las reflexiones de la $\mathrm{SiO}_{2}$ cristalina y de
After hydrothermal treatment in water, the intensity of the quartz peak rose, particularly after $200^{\circ} \mathrm{C}$ (compare Figures 1 (a) and (f)). The (BET-N $\mathrm{N}_{2}$ ) surface area of the untreated fly ash, R3, grew by up to three orders of magnitude after treatment, from 0.79 to $2.4 \mathrm{~m}^{2} / \mathrm{g}$ at $200^{\circ} \mathrm{C}$ (see Figure 2).

When the fly ash was hydrothermally treated in a $\mathrm{NaOH}$ medium, the main changes began to appear after $100^{\circ} \mathrm{C}$ (Fig 1 (d)-NaOH), when gismondine family zeolite $\mathrm{Na}-\mathrm{P} 1$ $\left(\mathrm{Na}_{6} \mathrm{Al}_{6} \mathrm{Si}_{10} \mathrm{O}_{32} \cdot 12 \mathrm{H}_{2} \mathrm{O}\right)$ formed. Here the surface area of the initial fly ash grew up to 13-fold: from 0.79 to 10.2 $\mathrm{m}^{2} / \mathrm{g}$ (Figure 2). At $150^{\circ} \mathrm{C}$ (Figure 1 (e), $\mathrm{NaOH}$ ), the amount of zeolite $\mathrm{Na}-\mathrm{P} 1$ rose, the amorphous $\mathrm{SiO}_{2}$ halo disappeared and the crystalline $\mathrm{SiO}_{2}$ and mullite peaks declined. At $200^{\circ} \mathrm{C}$, zeolite $\mathrm{Na}-\mathrm{P} 1$ was transformed into 
la mullita disminuyen. A $200^{\circ} \mathrm{C}$, la zeolita $\mathrm{Na}-\mathrm{P} 1$ se transforma en analcima-C $\left(\mathrm{Na}\left(\mathrm{Si}_{2} \mathrm{Al}\right) \mathrm{O}_{6} \mathrm{H}_{2} \mathrm{O}\right)$ y sodalita $\left(1.08 \mathrm{Na}_{2} \mathrm{O}_{2} \mathrm{Al}_{2} \mathrm{O}_{3} \cdot 1.68 \mathrm{SiO}_{2} .1 .8 \mathrm{H}_{2} \mathrm{O}\right)$; también se detectan trazas de tobermorita- $11 \AA\left(\mathrm{Ca}_{5}(\mathrm{OH})_{2} \mathrm{Si}_{6} \mathrm{O}_{16} \cdot 4 \mathrm{H}_{2} \mathrm{O}\right)$. A esta temperatura todos los compuestos de la ceniza volante de partida R3 han desaparecido, lo que sugiere su total transformación.

Esta transformación en zeolitas con sodio provoca un importante aumento del área superficial de la ceniza volante, llegando a ser 13 veces superior la de la ceniza de partida (Figura 2). analcime- $\mathrm{C} \quad\left(\mathrm{Na}\left(\mathrm{Si}_{2} \mathrm{Al}\right) \mathrm{O}_{6} \mathrm{H}_{2} \mathrm{O}\right)$ and sodalite $\left(1.08 \mathrm{Na}_{2} \mathrm{O} \cdot \mathrm{Al}_{2} \mathrm{O}_{3} .1 .68 \mathrm{SiO}_{2} \cdot 1.8 \mathrm{H}_{2} \mathrm{O}\right)$. Traces of tobermorite$1 \AA\left(\mathrm{Ca}_{5}(\mathrm{OH})_{2} \mathrm{Si}_{6} \mathrm{O}_{16} \cdot 4 \mathrm{H}_{2} \mathrm{O}\right)$ were also detected. All the initial $R 3$ fly ash compounds disappeared at this temperature, an indication that full transformation had taken place.

The transformations in sodium zeolites provoke a significant increase in surface area of fly ash, becoming 3 times higher than the starting fly ash (Figure 2).

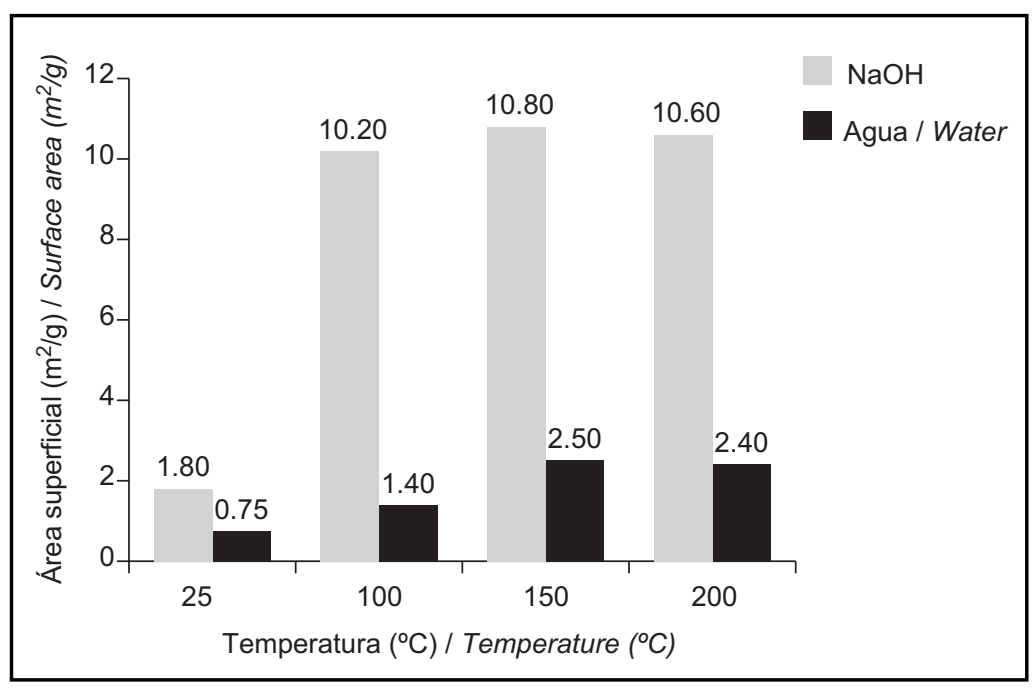

Figura 2. Evolución del área superficial de la ceniza volante en función de la temperatura del tratamiento hidrotermal en agua y en sosa.

Figure. 2. Evolution of surface area of fly ash with the temperature of hydrothermal treatment in water and $\mathrm{NaOH}$.

\subsection{Espectroscopía infrarroja por transformada de Fourier}

Los análisis de espectroscopia infrarroja por transformada de Fourier corroboran los resultados obtenidos mediante difracción de Rayos $\mathrm{X}$. Como se puede ver en la Figura 3, las principales bandas de absorción de la ceniza volante de partida que aparecen se atribuyen a la vibración del grupo $\mathrm{SiO}_{4}{ }^{4-}$ que presenta una banda ancha entre $800-1.400 \mathrm{~cm}^{-1}$ centrada en $1.068 \mathrm{~cm}^{-1}$, y a la vibración de los grupos $\mathrm{AlO}_{2}^{-}$que presenta bandas centradas en $450 \mathrm{~cm}^{-1}$ y $560 \mathrm{~cm}^{-1}$.

Estas bandas no sufren ningún cambio ni con el TH en agua a las diferentes temperaturas ni con el TH en medio alcalino a $\mathrm{T}^{\mathrm{a}}$ ambiente y a $50^{\circ} \mathrm{C}$; sin embargo, después de $100^{\circ} \mathrm{C}$ de $\mathrm{TH}$ en medio alcalino aparecen importantes cambios, que son descritos a continuación.

En la zona comprendida entre $800-500 \mathrm{~cm}^{-1}$ se aprecian modificaciones en las muestras sintetizadas a partir de

\subsection{Fourier transform infrared spectroscopy}

The Fourier transform infrared spectroscopic analyses corroborated the X-ray diffraction findings. As Figure 3 shows, the main absorption bands for the initial fly ash were attributed to the vibration in the $\mathrm{SiO}_{4}^{4-}$ group, which appeared as a wide band stretching from 800 to $1400 \mathrm{~cm}^{-1}$ and centred over $1068 \mathrm{~cm}^{-1}$, and to $\mathrm{AlO}_{2}^{-}$ group vibrations, with bands centred over 450 and $560 \mathrm{~cm}^{-1}$.

These bands remained unchanged after HT in water at any temperature as well as in the alkaline medium at ambient temperature and $50{ }^{\circ} \mathrm{C}$. After hydrothermal treatment at $100{ }^{\circ} \mathrm{C}$ in the alkaline medium, however, the following significant changes were observed.

In the samples synthesized at $100{ }^{\circ} \mathrm{C}$ or higher, changes were observed in the area from 800 to $500 \mathrm{~cm}^{-1}$, which 
$100^{\circ} \mathrm{C}$; que corresponden a las bandas de vibración de las unidades estructurales secundarias (SBU) de las zeolitas (hay un aumento en la intensidad de la banda de $450 \mathrm{~cm}^{-1}$ y aparecen dos nuevas bandas a $750 \mathrm{~cm}^{-1}$ y $619 \mathrm{~cm}^{-1}$ ); la intensidad de las bandas atribuidas a la vibración de las moléculas de agua que aparecen a 3.450 $\mathrm{cm}^{-1}, 1.650 \mathrm{~cm}^{-1}$ y $1.460 \mathrm{~cm}^{-1}$ aumenta considerablemente. En las muestras sintetizadas a $100^{\circ} \mathrm{C}$ y $150^{\circ} \mathrm{C}$, la banda ancha centrada a $1.068 \mathrm{~cm}^{-1}$ se desplaza a $1.000 \mathrm{~cm}^{-1}$ con un hombro a $1.100 \mathrm{~cm}^{-1}$, incluso aumentando su intensidad; A $200{ }^{\circ} \mathrm{C}$, probablemente debido a la transformación de zeolita Na-P1 en Analcima: desaparece el hombro de $1.100 \mathrm{~cm}^{-1}$, aumenta la banda de $450 \mathrm{~cm}^{-1}$ y las bandas de $750 \mathrm{~cm}^{-1}$ y $619 \mathrm{~cm}^{-1}$ disminuyen su intensidad. involved the zeolite secondary building unit (SBU) vibration bands: the intensity of the band at $450 \mathrm{~cm}^{-1}$ grew and two new bands appeared, at 750 and $619 \mathrm{~cm}^{-1}$. The intensity of the bands attributed to the vibration of water molecules appearing at 3450, 1650 and $1460 \mathrm{~cm}^{-1}$ rose substantially. In the samples synthesized at 100 and $150^{\circ} \mathrm{C}$, the wide band centred over $1068 \mathrm{~cm}^{-1}$ shifted to $1000 \mathrm{~cm}^{-1}$, "grew" a shoulder at $1100 \mathrm{~cm}^{-1}$ and even became more intense. At $200{ }^{\circ} \mathrm{C}$, very likely due to the transformation of zeolite Na-P1 into analcime, the shoulder at $1100 \mathrm{~cm}^{-1}$ disappeared, the band at $450 \mathrm{~cm}^{-1}$ grew and the intensity of the bands at 750 and $619 \mathrm{~cm}^{-1}$ declined.

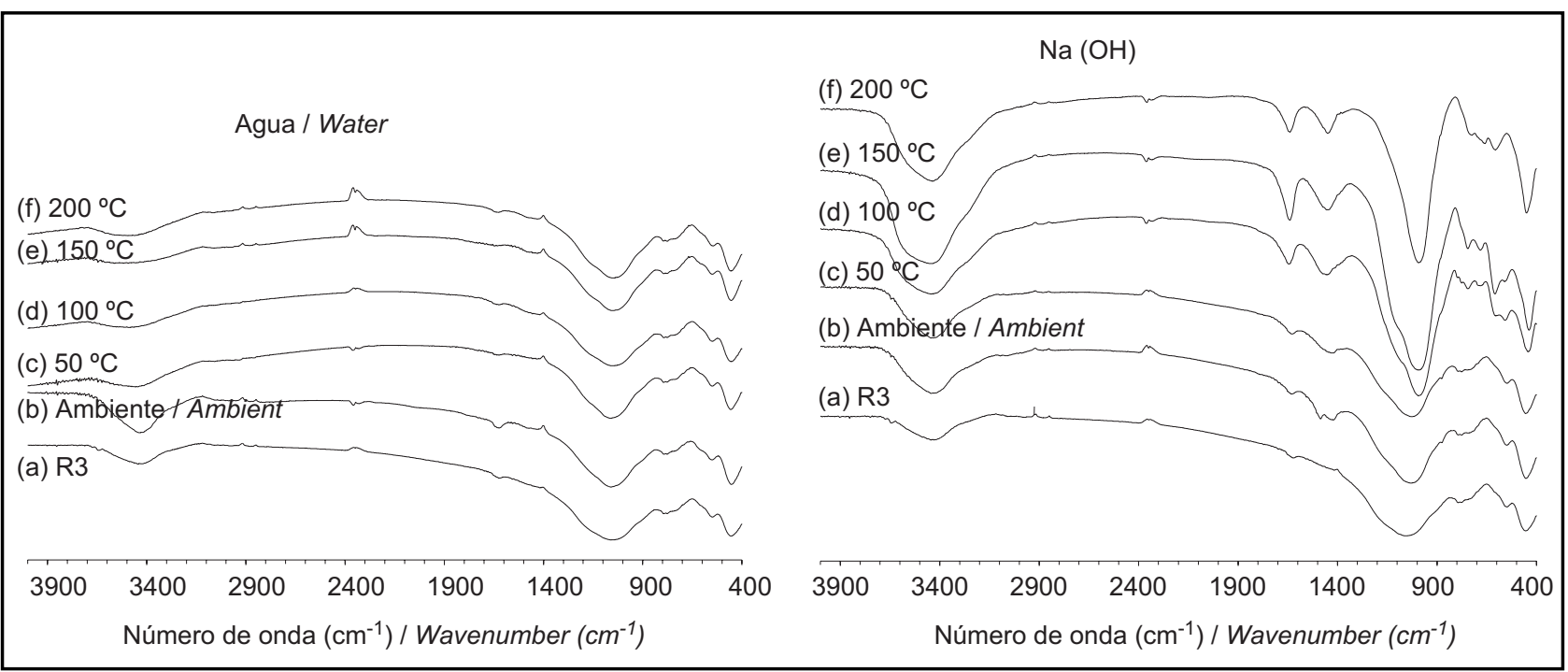

Figura 3. Espectros infrarrojos de la ceniza volante durante el tratamiento hidrotermal en agua y en disolución $\mathrm{Na}(\mathrm{OH}) 1 \mathrm{M}$. Figure 3. Changes of FTIR spectrum of fly ash during the hydrothermal treatment in water and $\mathrm{NaOH} 1 \mathrm{M}$ solution.

\subsection{Análisis térmico}

Las curvas termogravimétricas (TG/DTG) de las muestras más representativas junto con la evolución de la pérdida de peso con la temperatura del TH en ambos medios aparecen en la Figura 4. Como se muestra en la figura, las curvas de TG/DTG de la ceniza volante después del TH en agua a las diferentes temperaturas de tratamiento son prácticamente similares a las de la ceniza de partida (ver Figura 4 (a-b)). No se observan cambos significativos en la pérdida de peso de la ceniza volante.

En el caso de la disolución alcalina, los principales cambios ocurren desde los $100^{\circ} \mathrm{C}$. Hay un fuerte aumento en la pérdida de peso desde $50^{\circ} \mathrm{C}$ hasta los $150{ }^{\circ} \mathrm{C}$, que se atribuye a las moléculas de agua de los compuestos tipo zeolita, a dicha temperatura es cuando mejor se formaba la zeolita Na-P1 tipo gismondina. La cantidad de

\subsection{Thermal analysis}

The thermogravimetric (TG/DTG) curves for the most representative samples, together with the weight loss at rising $H T$ temperatures in both media, are shown in Figure 4. As the figure shows, the TG/DTG curves for the fly ash hydrothermally treated in water at the various temperatures were essentially similar to the curves for the initial ash (see Figure $4(a-b))$. No significant weight loss was observed in the fly ash.

In the alkaline solution, the major changes took place at temperatures of $100^{\circ} \mathrm{C}$ or over. A steep weight loss was recorded from 50 to $150{ }^{\circ} \mathrm{C}$, attributed to the water molecules in the zeolite-type compounds. This was the temperature at which gismondine group zeolite $\mathrm{Na}-\mathrm{P} 1$ formed most effectively. Further to the stoichiometry of 
agua, de acuerdo con la estequiometría de esta zeolita, representa un pérdida de peso del $16,5 \%$, valor que se obtiene a la temperatura de $150^{\circ} \mathrm{C}$ (Figura 4 (e)). Esto parece indicar que el $100 \%$ de la ceniza volante se ha convertido en zeolita Na-P1. La descomposición térmica de esta zeolita da lugar a dos picos centrados a $80^{\circ} \mathrm{C}$ y this zeolite, the water content accounted for a weight loss of $16.5 \%$, which was the value obtained at $150^{\circ} \mathrm{C}$ (Figure $4(\mathrm{e})$ ). This would appear to mean that $100 \%$ of the fly ash was converted into zeolite Na-P1. The thermal decomposition of this zeolite gave rise to two peaks, centred over 80 and $135^{\circ} \mathrm{C}$ (Figure 4 (c)). Weight loss
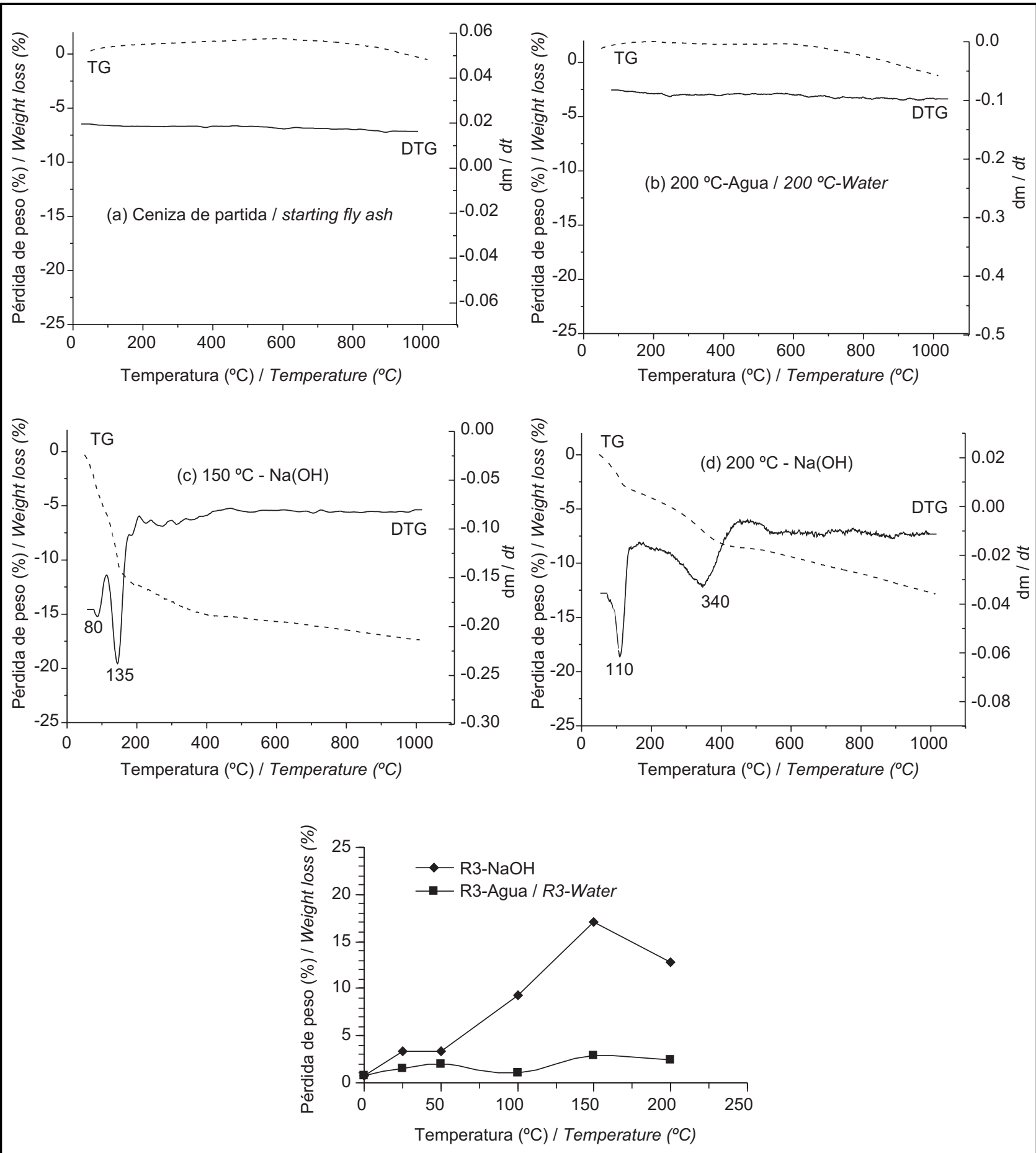

Figura 4. Curvas de TG y DTG de la ceniza volante (a-d) y pérdida de peso evaluado de la curva de TG a diferentes temperaturas del tratamiento hidrotermal.

Figure 4. Changes of TG and DTG curves of fly ash (a-d) and weight losses evaluated from TG at the different temperatures of hydrothermal treatments. 
$135^{\circ} \mathrm{C}$ (Figura 4 (c)). A $200^{\circ} \mathrm{C}$, se produce una caída en la pérdida de peso, que podría ser atribuida a la conversión de zeolita Na-P1 gismondina en analcima y sodalita, con una consecuente disminución del número de moléculas de agua (de acuerdo con sus estequiometrías). Además la correspondiente curva DTG muestra dos picos centrados en $110^{\circ} \mathrm{C}$ y $340^{\circ} \mathrm{C}$ (Figura 4(d)).

La conversión de zeolita Na-P1 gismondina $\left(\mathrm{Na}_{6} \mathrm{Al}_{6} \mathrm{Si}_{10} \mathrm{O}_{32}\right.$. $\left.12 \mathrm{H}_{2} \mathrm{O}\right)$, de estructura cristalina tetragonal, en analcima $\left(\mathrm{Na}\left(\mathrm{Si}_{2} \mathrm{Al}\right) \mathrm{O}_{6} \mathrm{H}_{2} \mathrm{O}\right)$ de estructura cúbica, es causada por el aumento de la temperatura del tratamiento hidrotermal desde $150^{\circ} \mathrm{C}$ a $200^{\circ} \mathrm{C}$. Probablemente, a la temperatura de $200^{\circ} \mathrm{C}$ se pierde agua estructural de la zeolita tipo gismondina que desestabiliza su red cristalina evolucionando hacia la fase cúbica más estable termodinámicamente. Esta hipótesis está avalada por los resultados de las pérdidas de peso termogravimétricas correspondientes a la volatilización de las moléculas de agua de la estructura de la zeolita, cuya evolución con la temperatura del tratamiento hidrotermal (Figura 4) muestra una disminución bastante considerable entre $150^{\circ} \mathrm{C}$ y $200^{\circ} \mathrm{C}$, así como un desplazamiento de la temperatura de volatilización del agua hacia valores mayores.

\section{CONCLUSIONES}

- Cuando el TH tiene lugar con agua, la ceniza volante no sufre cambios importantes.

- En el caso del TH con disolución $\mathrm{NaOH} 1 \mathrm{M}$, la zeolita tipo Na-P1 comienza a formarse a $100{ }^{\circ} \mathrm{C}$, aumentando a $150^{\circ} \mathrm{C}$.

- La conversión de zeolita tipo Na-P1 en zeolita analcime-C se produce a $200^{\circ} \mathrm{C}$, junto con otros compuestos minoritarios, como son la sodalita y la tobermorita.

- El área superficial de la ceniza volantes aumenta más de un orden de magnitud como resultado de la conversión en zeolita.

- La conversión de ceniza volante en zeolita mediante un $\mathrm{TH}$ a $100{ }^{\circ} \mathrm{C}$ tiene importantes aplicaciones en la inmovilización de especies con carácter tóxico.

\section{AGRADECIMIENTOS}

Los autores agradecen la financiación por parte de la Comisión Interministerial de Ciencia y Tecnología mediante la concesión del proyecto PB98-0516 para la realización de estos trabajos; así como a la central térmica de Soto de Rivera por el suministro de la ceniza volante. declined at $200{ }^{\circ} \mathrm{C}$, which may be attributed to the conversion of the zeolite $\mathrm{Na}-\mathrm{P} 1$ isotype of gismondine into analcime and sodalite, with the concomitant drop in the number of water molecules (according to the stoichiometry). The DTG curve also contained two peaks centred at 110 and $340{ }^{\circ} \mathrm{C}$ (Figure 4 (d)).

The conversion of gismondine zeolite $\mathrm{Na}-\mathrm{P} 1\left(\mathrm{Na}_{6} \mathrm{Al}_{6} \mathrm{Si}_{10} \mathrm{O}_{32}\right.$. $\left.12 \mathrm{H}_{2} \mathrm{O}\right)$, which has a tetragonal crystalline structure, into analcime $\left(\mathrm{Na}\left(\mathrm{Si}_{2} \mathrm{Al}\right) \mathrm{O}_{6} \mathrm{H}_{2} \mathrm{O}\right)$, which is cubic, occurred when the hydrothermal treatment temperature was raised from 150 to $200{ }^{\circ} \mathrm{C}$. At $200{ }^{\circ} \mathrm{C}$, the gismondinetype zeolite probably underwent structural water loss, resulting in the destabilization of its crystalline network, which would evolve toward the more thermodynamically stable cubic phase. Evidence for this hypothesis was provided by the thermogravimetric weight loss findings attributed to the volatilization of water molecules in the zeolite structure. The variation in weight loss with hydrothermal treatment temperature (Figure 4) showed a fairly substantial decline from 150 to $200{ }^{\circ} \mathrm{C}$, along with a shift in the water volatilization temperature to higher values.

\section{CONCLUSIONS}

- When HT is conducted in water, the fly ash undergoes no significant change.

- Where the HT medium is $1-\mathrm{M} \mathrm{NaOH}$, zeolite $\mathrm{Na}-\mathrm{P} 1$ begins to form at $100{ }^{\circ} \mathrm{C}$, with a visible increase at $150^{\circ} \mathrm{C}$.

- Zeolite Na-P1 converts to zeolite analcime-C and other minority compounds such as sodalite and tobermorite at $200{ }^{\circ} \mathrm{C}$.

- The surface area of fly ash grows by more than one order of magnitude as a result of conversion into zeolite.

- The conversion of fly ash into zeolite via HT at $100^{\circ} \mathrm{C}$ has a number of practical applications in terms of immobilizing toxic species.

\section{ACKNOWLEDGEMENTS}

This research was funded by the Interministerial Commission for Science and Technology under project PB98-0516. The authors wish to thank the Soto de Ribera steam power plant for supplying the fly ash used in the experiments. 


\section{BIBLIOGRAFÍA / BIBLIOGRAPHY}

(1) Guerrero, A.; Goñi, S.; Campillo, I.; and Moragues, A.: "Belite Cement Clinker from Coal Fly Ash of High Ca Content. Optimization of Synthesis Parameters", Environ. Sci. Technol., vol. 38 (2004), pp. 3209-3213. doi:10.1021/es0351589

(2) Goñi, S.; Guerrero, A.; Luxán, M. P. and Macías, A.: "Dehydration of Pozzolanic Products Hydrothermally Synthetized from Fly Ashes", Mater. Res. Bull., vol. 35 (2000), pp. 1333-1344. doi:10.1016/S0025-5408(00)00326-3

(3) Guerrero, A.; Fernández, E.; Macías, A. and Goñi, S.: "Hydrothermal Treatment of Fly Ash from Municipal Solid Waste Incineration", in Proc. of Waste Materials in Construction: Science and Engineering of Recycling for Environmental Protection, edited by G.R. Woolley, J.J.M. Goumans and P.J. Wainwright. Pergamon, Amsterdam (2000), pp. 178-185.

(4) Guerrero, A.; Goñi, S.; Macías, A.; Luxán, M. P.: "Hydraulic Activity and Microstructural Characterization of New Fly Ash-Belite Cements Synthesized at Different Temperatures". J. Mater. Res., vol. 14, (6) (1999), pp. 2680-2687. doi:10.1557/JMR.1999.0359

(5) Gutiérrez, P.; Guerrero, A.; Goñi, S.; Lorenzo, M. P. and Macías, A.: (2002) "Development of a Low Energy Environmentally Friendly Cement" in Sustainable Concrete Construction. Edit. Ravindra K. Dhir, Thomas D. Dyer and Judith E. Halliday, Publishers: Thomas Telford, pp. 95-106.

(6) Goñi, S.; Guerrero, A.; Moragues, A.; Tallafigo, M. F.; Campillo, I.; Sánchez, J. and Porro, A.: (2003): "New Belite Cement Clinkers from Fly ash of Coal Combustion of High Ca Content". Spanish Patent No ES222 3275 (IPC: C04B7/04).

(7) Peña Penilla, R.; Guerrero, A.; Goñi Elizalde, S.: "Zeolite synthesized by alkaline hydrothermal treatment of bottom ash from combustion of municipal solid wastes", J. Am. Ceram. Soc., vol. 86(9) (2003), pp. 1527-1533. doi:10.1111/j.1151-2916.2003.tb03509.x

(8) Peña, R.; Guerrero, A. and Goñi, S.: "Hydrothermal Treatment of Bottom Ash from the Incineration of Municipal Solid Waste: Retention of Cs(I), Cd (II), Pb (II) and Cr (III)", J. Hazard. Mater., vol. B129, (2006), pp. 151-157.

(9) Brough, A. R.; Katz, A.; Sun, G. K.; Struble, L. J.; Kirkpatrick, R. J.; Young, J. F.: "Adiabatically cured, alkali-activated cement-based wasteforms containing high levels of fly ash: Formation of zeolites and Al-substituted C-S-H", Cem. Concr. Res., vol. 31, (2001), p. 1437. doi:10.1016/S0008-8846(01)00589-0

(10) Brough, A. R.; Katz, A.; Bakharev, T.; Sun, G.-K.; Kirkpatrick, R. J.; Struble, L. J.; Young, J. F.: Mater. Res. Soc. Symp. Proc., 370, (1995), p. 199.

(11) Atkins, M.; Glasser, F. P.; Jack, J. J.: "Zeolite P in cements: its potential for immobilizing toxic and radioactive waste species", Waste Manag., vol. 15 (2) (1995), p. 127. doi:10.1016/0956-053X(95)00015-R

(12) LaRosa, J.; Kwan, S.; Glutzeck, M.W.: Mater. Res. Soc. Symp. Proc., 245, (1991), p. 211.

(13) Grutzeck, M.; Kwan, S.; DiCola, M.: "Zeolite formation in alkali-activated cementitious systems". Cem. Concr. Res., vol. 34 (6) (2004), pp. 949-955. doi:10.1016/j.cemconres.2003.11.003

(14) Canpolat, F.; Yilmaz, K.; Köse, M. M.; Sümer, M.; Yurdusev, MA.: (in press) "Use of zeolite, coal bottom ash and fly ash as replacement materials in cement production", Cem. Concr. Res., vol. 34 (5) (2004), pp. 731-735. doi:10.1016/S0008-8846(03)00063-2

(15) Ma, W.; Brown, P. W.; Komarneni, S.: "Characterization and cation exchange properties of zeolites synthesized from fly ashes", J. Mater. Res., vol. 13 (1) (1998), p. 3. doi:10.1557/JMR.1998.0001

(16) Querol, X.; Umaña, J.C.; Plana, F.; Alastuey, A.; López-Soler, A.; Medinaceli, A.; Valero, A.; Domingo, M J.; García-Rojo, E.: "Synthesis of zeolites from fly ash at pilot plant scale. Examples of potential applications", Fuel, vol. 80 (2001), pp. 857-865. doi:10.1016/S00162361(00)00156-3

(17) Querol, X.; Plana, F.; Alastuey, A.; López-Soler, A.: "Synthesis of Na-zeolites from fly ash", Fuel, vol. 76 (8) (1997), p. 793. doi:10.1016/S0016-2361(96)00188-3

(18) Moreno, N.; Querol, X.; Ayora, C.; Fernández-Pereira, C.; Janssen-Jurkovicová, M.: "Utilization of Zeolites Synthesized from Coal Fly Ash for the Purification of Acid Mine Waters", Environ. Sci. Technol. vol. 35(17) (2001), pp. 3526-3534. doi:10.1021/es0002924

(19) Moreno, N.; Querol, X.; Ayora, C.; Alastuey, A.; Fernández-Pereira, C.; Janssen-Jurkovicová, M.: "Potential Environmental Applications of Pure Zeolitic Material Synthesized from Fly Ash", J. Environ. Engineering, vol. 127(11) (2001), pp. 994-1002. doi:10.1061/(ASCE)07339372(2001)127:11(994)

(20) Grutzeck, M.W.; Siemer, D.D.: "Zeolites synthesized from class F fly ash and sodium aluminate slurry", J. Am. Ceram. Soc., vol. 80 (9) (1997), pp. 2449-2453.

(21) LaRosa, J.; Kwan, S.; Grutzeck, M.W.: "Zeolite formation in class F fly ash blended cement pasters", J. Am. Ceram. Soc., vol. 75 (1992), pp.1574-1580. doi:10.1111/j.1151-2916.1992.tb04228.x

(22) Amrhein, C.; Haghnia, G.H.; Kim, T.S.; Mosher, P.A.; Cgagajena, R.C.; Amanios, T.; De la Torre, L.: "Synthesis and properties of zeolites from coal fly ash", Environ. Sci. Technol. vol. 30 (3) (1996), pp. 735-742. doi:10.1021/es940482c

(23) Murayama, N.; Yamamoto, H.; Shibata, J.: Int. J. Miner. Process. vol. 64 (2002), p. 1. doi:10.1016/S0301-7516(01)00046-1

(24) Hollman, G.G.; Steenbruggen, G.; Janssen-Jurkovicová, M.: "A two-step process for the synthesis of zeolites from coal fly ash", Fuel, vol. 78 (1999), p. 1225. doi:10.1016/S0016-2361(99)00030-7

(25) Tanaka, H.; Sakai, Y.; Hino, R.: "Formation of Na-A and -X zeolites from waste solutions in conversion of coal fly ash to zeolites", Mat. Res. Bull., vol. 37 (11) (2002), pp. 1873-1884. doi:10.1016/S0025-5408(02)00861-9 